BULLETIN OF THE

AMERICAN MATHEMATICAL SOCIETY

Volume 77, Number 5, September 1971

\title{
VARIETIES OF LOCALLY CONVEX TOPOLOGICAL VECTOR SPACES
}

\author{
BY JOSEPH DIESTEL, SIDNEY A. MORRIS, STEPHEN A. SAXON
}

Communicated by Victor Klee, April 14, 1971

1. Introduction. A variety is defined here to be a nonempty class of real Hausdorff locally convex spaces (LCS's) closed under the operations of taking subspaces (not necessarily closed), separated quotients, arbitrary products and isomorphic images. The two extreme examples of a variety are the class of all LCS's and the class of all zero-dimensional LCS's. Less obvious examples are:

(a) the class of all Schwartz LCS's [8],

(b) the class of all nuclear LCS's [26],

(c) the class of all LCS's having their weak topology [8].

The potency of an analogous concept for groups [21] has manifested itself for three decades, and for topological groups has quite recently been asserted [3], [14]-[20]. In this note we announce selected results from a forthcoming paper [5] which, we hope, will convince the reader that the theory of varieties not only is of intrinsic interest, but lends to locally convex spaces a new and illuminating perspective which consolidates, strengthens and adds to significant parts of the literature.

2. Results. For any class $\mathcal{C}$ of LCS's, the variety generated by $\mathfrak{C}$, denoted by $\mathcal{U}(\mathfrak{C})$, is the smallest variety containing $\mathcal{C}$. For example, the variety generated by the class of all real Banach spaces is the class of all LCS's [26].

Consider these seven properties for Banach spaces: (i) reflexivity; (ii) quasi-reflexivity [4]; (iii) almost reflexivity [10]; (iv) separability; (v) having separable dual; (vi) being Hilbertian; (vii) having Hamel dimension $\langle\boldsymbol{N}$, where $\boldsymbol{N}$ is some fixed infinite cardinal. Theorem 1 is a stronger statement than the usual ones about closed subspaces, separated quotients and finite products of Banach spaces with one of the above properties.

AMS 1970 subject classifications. Primary 46A05; Secondary 46E10, 46E30, 46A45, 46M99.

Key words and phrases. Products, subspaces and quotients of locally convex spaces, Banach spaces, Fréchet spaces, nuclear spaces, Schwartz spaces, reflexivity, separability, weak topology, strongest locally convex topology, $l_{1}(\Gamma), l_{p}, L_{p}, c_{0}$, spaces of continuous functions, singly generated variety, universal generator.

Copyright (C) American Mathematical Society 1971 
THEOREM 1. If B is a class of Banach spaces all of which possess a given one of the above properties (i)-(vii), then each Banach space $E$ in $V(B)$ possesses the same property.

COROLLARY 2. The class of all varieties is not a set.

The variety generated by the class of all real $\boldsymbol{\aleph}_{0}$-dimensional normed spaces contains no infinite-dimensional Banach space. However,

THEOREM 3. The variety generated by an arbitrary class of Frechet spaces contains the completion of each of its members.

If $\mathcal{C}$ consists of the single LCS $E$, then the variety it generates is singly generated and denoted by $V(E)$.

THEOREM 4. A variety is singly generated if and only if it is contained in $\mathcal{V}\left(l_{1}(\Gamma)\right)$ for some set $\Gamma$.

CoROLlary 5. Any subvariety of a singly generated variety is singly generated.

The analogues to Corollaries 2 and 6 are false for groups [21].

COROLLARY 6. Not every variety is singly generated.

If a variety $v$ contains an LCS $E$ such that each LCS in $v$ is isomorphic to a subspace of a product of copies of $E$, then we say that $E$ is a universal generator for $v$.

THEOREM 7. Every singly generated variety has a universal generator.

Since every separable Banach space is a quotient of $l_{1}$, it follows that every separable LCS is contained in $v\left(l_{1}\right)$. In fact, $C([0,1])$ is a universal generator for $V\left(l_{1}\right)$. (Cf. [12].) Statement (9), \$2 of [27] implies that every Schwartz space is a subspace of a product of separable normed spaces. Thus we have proved the theorem of A. Todd, that $V\left(l_{1}\right)$ contains the variety of Schwartz spaces. Using Theorems 4 and 7 and Corollary 5 we obtain

THEOREM 8. The variety of Schwartz spaces, and hence also the variety of nuclear spaces, has a universal generator.

A deep result of Kömura and Kōmura [9] is that $(s)$ is a universal generator for the variety of nuclear spaces, where $(s)$ is the Fréchet space of rapidly decreasing sequences. (To our knowledge, no one has found a correspondingly serviceable and concrete universal generator

1 To appear in Mr. Todd's dissertation at the University of Florida. 
for Schwartz spaces.) Using this result and [2], it is possible to generalize a theorem of Grothendieck so as to imply Theorem 9 below, [25]. Grothendieck's original version ([7], also [26, p. 101]) implies Theorem 9 for the special case $E=l_{p}(1 \leqq p \leqq \infty)$.

THEOREM 9. Let $E$ be an arbitrary infinite-dimensional Banach space. Then $V(E)$ contains the variety of nuclear spaces. Indeed, each nuclear space is isomorphic to a subspace of a product of copies of $E$.

Theorem 9 indicates that the variety of nuclear spaces is a relatively small one. In regard to absolute smallness we have

THEOREM 10. (i) The variety of all LCS's with their weak topology has $R$ (the reals) as a universal generator, and hence is the smallest nontrivial variety.

(ii) There exists a (unique) second smallest variety $v_{\neq} \mathcal{V}(R)$, in the sense that every variety properly containing $V(R)$ must contain $V$. $V$ has $\varphi$ as a universal generator, where $\varphi$ is an $\boldsymbol{\aleph}_{0}$-dimensional real vector space given the strongest locally convex topology [24].

(iii) There exists no third smallest variety.

Corollary 11. An LCS $E$ has its weak topology if and only if $v(E)$ does not contain $\varphi$.

Also of interest with regard to size is

THEOREM 12. Any variety generated by an infinite-dimensional normed space contains a maximal proper subvariety.

Finally, we investigate the varieties generated by the well-known Banach spaces. Using Theorem 1 and results in [1], [7], [13] and [22], we obtain

TheOREM 13. For $1<p<\infty, K$ any uncountable compact metric space and $(s)$ the Frechet space of rapidly decreasing sequences of [9],

$$
\begin{aligned}
v(R) & \varsubsetneqq v(\varphi) \varsubsetneqq v((s)) \varsubsetneqq v\left(l_{p}\right) \subseteq\left(v L_{p}\right) \\
& \varsubsetneqq v\left(l_{1}\right)=v(C(K))=v\left(L_{1}\right) \varsubsetneqq v\left(l_{\infty}\right)=v\left(L_{\infty}\right) .
\end{aligned}
$$

A classical result of Banach [1] says that if $1<p \neq q<\infty$ then $l_{p}$ is not isomorphic to a subspace of $l_{q}$; in fact it follows from

THEOREM 14. $l_{p} \notin U\left(l_{q}\right)$ for $1<p \neq q<\infty$.

That $l_{p}$ cannot be manufactured from $l_{q}$ using (repeatedly) the operations of taking subspaces, quotients and products.

Since $l_{2}$ is a subspace of $L_{p}$ for $1 \leqq p \leqq \infty$ [28], we have

Corollary 15. $v\left(l_{p}\right) \varsubsetneqq v\left(L_{p}\right)$ for $1<p<\infty, p \neq 2$. 
Recently, the problem of Banach's linear dimension for $L_{p}$ has been completely solved [11]. The stronger varietal statement is

THEOREM 16. Let $1<p \neq q<\infty$. Then the following are equivalent:

(i) $L_{p} \in \mathcal{V}\left(L_{q}\right)$,

(ii) $l_{p} \in \mathcal{V}\left(L_{q}\right)$,

(iii) $q<p \leqq 2$ or $2 \leqq p<q$.

By Theorem 1(iii), every Banach space in $U\left(c_{0}\right)$ is almost reflexive. Surprisingly we have, by using the results of [6],

THEOREM 17. $\mathcal{V}\left(c_{0}\right)$ contains no infinite-dimensional reflexive Banach space. Indeed, no weakly sequentially complete Banach space is in $\mathrm{V}\left(c_{0}\right)$.

REMARK. By Theorems $1(\mathrm{i}), 3$ and 17 we see that $\mathcal{V}\left(c_{0}\right) \cap \mathcal{V}\left(l_{p}\right)$, for $1<p<\infty$, is a variety which contains no infinite-dimensional normed spaces. This emphasizes, by Theorem 9, that no infinite-dimensional normed space is nuclear.

In conclusion we mention the

THEOREM 18. Let $X$ and $Y$ be compact Hausdorff spaces. If $X$ is dispersed [23] and $Y$ is not dispersed, then $C(Y) \notin v(C(X))$.

\section{REFERENCES}

1. S. Banach, Théorie des opérations linéaires, Monografie Mat., PWN, Warsaw, 1932.

2. C. Bessaga and A. Pelczyński, On bases and unconditional convergence of series in Banach spaces, Studia Math. 17 (1958), 151-164. MR 22 \#5872.

3. M. S. Brooks, S. A. Morris and S. A. Saxon, Generating varieties of topological groups, Proc. Amer. Math. Soc. (to appear).

4. P. Civin and B. Yood, Quasi-reflexive spaces, Proc. Amer. Math. Soc. 8 (1957), 906-911. MR 19, 756.

5. J. Diestel, S. A. Morris and S. A. Saxon, Varieties of linear topological spaces, Trans. Amer. Math. Soc. (to appear).

6. A. Grothendieck, Sur les applications linéaires faiblement compactes d'espaces $d u$ type $C(K)$, Canad. J. Math. 5 (1953), 129-173. MR 15, 438.

7. - Produits tensoriels topologiques et espaces nucleaires, Mem. Amer. Math. Soc. No. 16 (1955). MR 17, 763.

8. J. Horváth, Topological vector spaces and distributions. Vol. I, Addison-Wesley, Reading, Mass., 1966. MR 34 \#4863.

9. T. Kömura and Y. Kōmura, Über die Einbettung der nuklearen Räume in $(s)^{A}$, Math. Ann. 162 (1965/66), 284-288. MR 32 \#6190.

10. E. Lacey and R. J. Whitley, Conditions under which all the bounded linear maps are compact, Math. Ann. 158 (1965), 1-5. MR 30 \#3373.

11. J. Lindenstrauss and A. Pelczyński, Absolutely summing operators in $\mathfrak{L}_{p}$-spaces and their applications, Studia Math. 29 (1968), 275-326. MR 37 \#6743.

12. R. H. Lohman, An embedding theorem for separable locally convex spaces, Canad. Math. Bull. (to appear). 
13. A. A. Miljutin, Isomorphism of the spaces of continuous functions over compact sets of the cardinality of the continuum, Teor. Funkcir Funkcional. Anal. i Priložen. Vyp. 2 (1966), 150-156. (Russian) MR 34 \#6513.

14. S. A. Morris, Varieties of topological groups, Bull. Austral. Math. Soc. 1 (1969), 145-160. MR 41 \#3655a.

15. - Varieties of topological groups. II, Bull. Austral. Math. Soc. 2 (1970), 1-13. MR 41 \#3655b.

16. - Varieties of topological groups. III, Bull. Austral. Math. Soc. 2 (1970), 165-178. MR $41 \# 3655$ c.

17. - Varieties of topological groups. IV (to appear).

18. - Free products of topological groups, Bull. Austral. Math. Soc. 4 (1971), 17-29.

19. - - Free compact abelian groups (to appear).

20. - Varieties of topological groups, Bull. Austral. Math. Soc. 3 (1970), 429-431.

21. H. Neumann, Varieties of groups, Ergebnisse der Mathematik und ihrer Grenzgebiete, Band 37, Springer-Verlag, Berlin and New York, 1967. MR 35 \#6734.

22. A. Pelczynski, On the isomorphism of the spaces $m$ and $M$, Bull. Acad. Polon. Sci. Sér. Sci. Math. Astr. Phys. 6 (1958), 695-696. MR 21 \#1513.

23. A. Pelczyniski and Z. Semadeni, Spaces of continuous functions. III. Spaces $C(\Omega)$ for $\Omega$ without perfect subsets, Studia Math. 18 (1959), 211-222. MR 21 \#6528.

24. S. A. Saxon, Nuclear and product spaces, Baire-like spaces, and the strongest locally convex topology, Math. Ann. (to appear).

25. - Embedding nuclear spaces in products of an arbitrary Banach space, Proc. Amer. Math. Soc. (to appear).

26. H. H. Schaefer, Topological vector spaces, Macmillan, New York, 1966. MR 33 \#1689.

27. T. Terzioğlu, On Schwartz spaces, Math. Ann. 182 (1969), 236-242. MR 40 \#683.

28. A. Zygmund, Trigonometric series. Vol. 1, 2nd rev. ed., Cambridge Univ. Press, New York, 1959. MR 21 \#6498.

University of Florida, Gainesville, Florida 32601 